\title{
Procalcitonin is more likely to be released by the fetus rather than placental tissue during chorioamnionitis
}

\author{
Zbynek Stranak $^{\mathrm{a}, \mathrm{b}}$, Jaroslav Feyereis ${ }^{\mathrm{a}, \mathrm{b}}$, Peter Korcek ${ }^{\mathrm{a}, \mathrm{b}}$, Simona Feyereislova ${ }^{\mathrm{a}, \mathrm{b}}$, Ladislav Krofta ${ }^{\mathrm{a}, \mathrm{b}}$
}

\begin{abstract}
Aims. To analyze the relationship between maternal, cord blood and neonatal procalcitonin (PCT) levels in preterm deliveries with and without histologically proven chorioamnionitis (HCA).

Methods. 91 mother-infant pairs from 24+0 to 33+0 gestational weeks were analyzed. Procalcitonin was measured in all mothers within 24 hours before and subsequently in cord blood and in neonates within the first two hours after delivery. PCT levels were analysed in relationship to HCA and clinical outcome.

Results. HCA was confirmed in 28 cases (31\%). We found no differences in PCT values between HCA positive and negative groups in maternal blood $(0.1 \pm 0.1 \mathrm{vs} 0.09 \pm 0.09 \mathrm{ng} / \mathrm{L}, P=0.76)$. PCT values in cord blood and neonates were significantly higher in the HCA positive compared to HCA negative group $(0.23 \pm 0.1 \mathrm{vs} 1.2 \pm 2.7 \mathrm{ng} / \mathrm{L}, P<0.001$ and $0.89 \pm 3.4$ vs $4.2 \pm 9.3 \mathrm{ng} / \mathrm{L}, P<0.0001$ respectively). PCT values in neonates were significantly higher than those of cord blood. Levels were not influenced by the mode of delivery, gestational age or premature rupture of membranes. Chorioamnionitis was more frequently associated with early onset neonatal sepsis (36\% in HCA group vs $5 \%$ in non HCA group, $P<0.0001)$. Comparison of other clinical data revealed no differences between HCA positive and negative groups. Conclusion. This study showed higher PCT in cord and neonatal blood in the presence of proven histological chorioamnionitis. The measurement of PCT in mothers' blood is not helpful for diagnosis of HCA. The changes in PCT values shown suggest its production and release by fetal tissue.
\end{abstract}

Key words: procalcitonin, preterm labor, intra-amniotic infection, early onset sepsis

Received: May 24, 2016; Accepted: July 29, 2016; Available online: September 5, 2016

http://dx.doi.org/10.5507/bp.2016.041

${ }^{a} 3^{r d}$ Faculty of Medicine, Charles University in Prague, Czech Republic

'Institute for the Care of Mother and Child, Prague, Czech Republic

Corresponding author:Peter Korcek, e-mail: peterkorcek.upmd@gmail.com

\section{INTRODUCTION}

Preterm birth represents a serious challenge for perinatal medicine which is reflected in its contribution to over $70 \%$ of perinatal mortality in developed countries. In addition, surviving infants frequently suffer from cardiorespiratory problems, mental retardation, cerebral palsy, and vision and hearing impairment ${ }^{1}$.

The most common cause of preterm birth is chorioamnionitis which is implicated in the majority of extremely preterm births. Chorioamnionitis is often asymptomatic and clinical signs lack both sensitivity and specificity over one third of women with these signs do not have histological evidence of placental inflammation as other causes may produce similar clinical features to chorioamnionitis $^{2,3}$.

Chorioamnionitis can occur without microbiologically-proven amniotic fluid infection in over half of the cases. However, released endotoxins and exotoxins possibly by bacteria colonizing the choriodecidual space initiate production of inflammatory cytokines provoking the maternal and fetal inflammatory response syndrome ${ }^{4}$.

Procalcitonin (PCT) is a peptide pro-hormone of calcitonin, physiologically produced by the thyroid gland. In response to endotoxin and inflammatory cytokines, PCT can be produced by monocytes and other organs ${ }^{2,5}$.
In contrast to $\mathrm{C}$-reactive protein (CRP), PCT reflects the onset and resolution of the inflammatory process more accurately. Thus, PCT may be more useful than CRP in the early diagnosis of infection and monitoring of the disease ${ }^{2,3}$.

For this reason, PCT plays an important role in the diagnosis of neonatal sepsis despite the fact that it may be elevated in non-infected infants with other complications ${ }^{2}$. PCT is also increased in cord blood of chorioamnionitisaffected newborns. Furthermore, the measurement of PCT in the maternal blood remains controversial in the diagnosis of intra-amniotic infection and chorioamnionitis $^{5,6}$.

The aim of our study was to investigate the relationship between histologically proven chorioamnionitis and maternal, umbilical and neonatal PCT levels.

\section{MATERIALS AND METHODS}

This retrospective study was approved by the Local Ethics Committee and the Local Committee on Human Research. Inclusion criteria were: preterm delivery at $24+0$ to $33+0$ weeks of gestation, patients born in our perinatal centre, completed measurements of the inflammatory parameters in the mother within $24 \mathrm{~h}$ before de- 
livery, completed measurement of procalcitonin (PCT) in the newborn within $2 \mathrm{~h}$ after delivery, completed measurement of procalcitonin in the cord blood and completed histological examination of the placenta. Excluded were all patients with incomplete biochemical, haematological and histological investigations, patients with severe congenital anomalies, patients who required resuscitation at the delivery room and patients with unknown outcome. Between January 2005 and December 2014, 91 motherinfant pairs were eligible for the study.

\section{Blood sampling and tests}

Blood samples for the assessment of PCT, C-reactive protein (CRP) and white blood count (WBC) were taken from the mother within 24 hours before preterm delivery (venous blood) and subsequently from the newborn within the first two hours after delivery (venous or arterial blood). Cord blood was obtained immediately after delivery from the umbilical vein. Blood counts were measured with a Coulter Micro Dif II (Coulter Electronics Ltd., Fullerton, US) and CRP was measured by turbidimetry (SLT Spectra, Austria). Immunoluminometric assay (Lumitest PCT, Brahms, Germany) was used for PCT analysis. Luminescence was measured automatically in a Berilux Analyser (Behring Diagnostics, Germany).

\section{Clinical and histological chorioamnionitis}

Clinical chorioamnionitis was defined using criteria proposed by $\mathrm{Gibbs}^{7}$ : body temperature $\geq 37.8{ }^{\circ} \mathrm{C}$ and a minimum of two other criteria: maternal or fetal tachycardia, uterine tenderness, malodorous vaginal discharge and leucocytosis $\geq 15000 \mathrm{~mm}^{-3}$. Histologically proven chorioamnionitis was defined by the presence of at least one inflammatory change in the placenta (intervillositis, deciduitis, or villositis), in membranes (amnionitis, deciduitis, or chorioamnionitis), or in the umbilical cord (funiculitis).

\section{Postnatal data}

Data were collected from the patient medical records. The definition of early-onset sepsis was based on the criteria proposed by Chiesa $^{6}$ (neonates with a positive blood culture and clinical signs of infection and/or neonates with negative blood culture, clinical signs of infection and positive sepsis screen). Other neonatal outcomes (respiratory distress syndrome, patent ductus arteriosus, intraventricular hemorrhage, necrotizing enterocolitis, periventricular leukomalacia and bronchopulmonary dysplasia) were followed up according to the VermontOxford definition ${ }^{8}$.

\section{Statistical analysis}

Data are reported using descriptive statistical methods. Univariate analyses were performed using Chi-square, Fisher's exact and Mann-Whitney U tests. All reported $P$-values are two-sided and not adjusted for multiplicity. $P<0.05$ was considered statistically significant. Data analysis was performed using the IBM SPSS Statistics 23.0.0.0 software (IBM Corp., Armonk, NY).

\section{RESULTS}

The study population consisted of 91 mother-infant pairs. The mean gestational age of the study group was 28.9 weeks (range 24 - 33 weeks). Premature preterm rupture of membranes (PPROM) was recorded in 50 cases $(55 \%)$ with average duration $24 \mathrm{~h}$ (range 2 - 136 hrs). No case of clinical chorioamnionitis was found. In contrast, histological chorioamnionitis was confirmed in 28 cases $(31 \%)$.

\section{Diagnosis of chorioamnionitis from maternal blood}

We found no significant differences in procalcitonin values between histologically chorioamnionitis positive and negative groups in maternal blood. The measurement of WBC and CRP was more accurate in detection of subclinical chorioamnionitis. The incidence of chorioamnionitis was not influenced by the PPROM. The results are shown in Table 1.

\section{PCT value in the diagnosis of chorioamnionitis}

The measurement of PCT in maternal blood disclosed very low PCT values and there was no difference between "chorioamnionitis" and "no chorioamnionitis" groups. The comparison of PCT level in cord blood revealed the association with chorioamnionitis (no chorioamnionitis: $0,23 \pm 0.1 \mathrm{ug} / \mathrm{L}$ vs. $1.2 \pm 2.7 \mathrm{ug} / \mathrm{L}$ in chorioamnionitis group, $P<0.0001)$. PCT values were significantly raised and higher in preterm neonates following pregnancies complicated by chorioamnionitis. The changes of PCT values in maternal, cord and neonatal blood are demonstrated in Fig. 1.

Table 1. Maternal inflammatory parameters in study population and as per study group (no chorioamnionitis versus histologically proven chorioamnionitis).

\begin{tabular}{|c|c|c|c|c|}
\hline & $\begin{array}{c}\text { Study group } \\
\mathrm{n}=91\end{array}$ & $\begin{array}{l}\text { No chorioamnionitis group } \\
n=63\end{array}$ & $\begin{array}{l}\text { Chorioamnionitis group } \\
\mathrm{n}=28\end{array}$ & $P$ \\
\hline $\begin{array}{l}\text { WBC count }(\mathrm{x} 1000 / \mathrm{uL}) \\
\text { mean }+(95 \% \mathrm{CI})\end{array}$ & $13.8(13.0-14.6)$ & $13.1(12.2-14.0)$ & $16.0(14.6-17.5)$ & 0.001 \\
\hline $\begin{array}{l}\text { C-reactive protein }(\mathrm{mg} / \mathrm{L}) \\
\text { mean }+(95 \% \mathrm{CI})\end{array}$ & $17.5(11.8-23.2)$ & $8.4(4.9-11.9)$ & $38.8(24.0-53.6)$ & 0.0001 \\
\hline $\begin{array}{l}\text { Procalcitonin }(\mathrm{ug} / \mathrm{L}) \\
\text { mean }+(95 \% \mathrm{CI})\end{array}$ & $0.10(0.07-0.12)$ & $0.1(0.07-0.13)$ & $0.09(0.05-0.11)$ & 0.76 \\
\hline
\end{tabular}




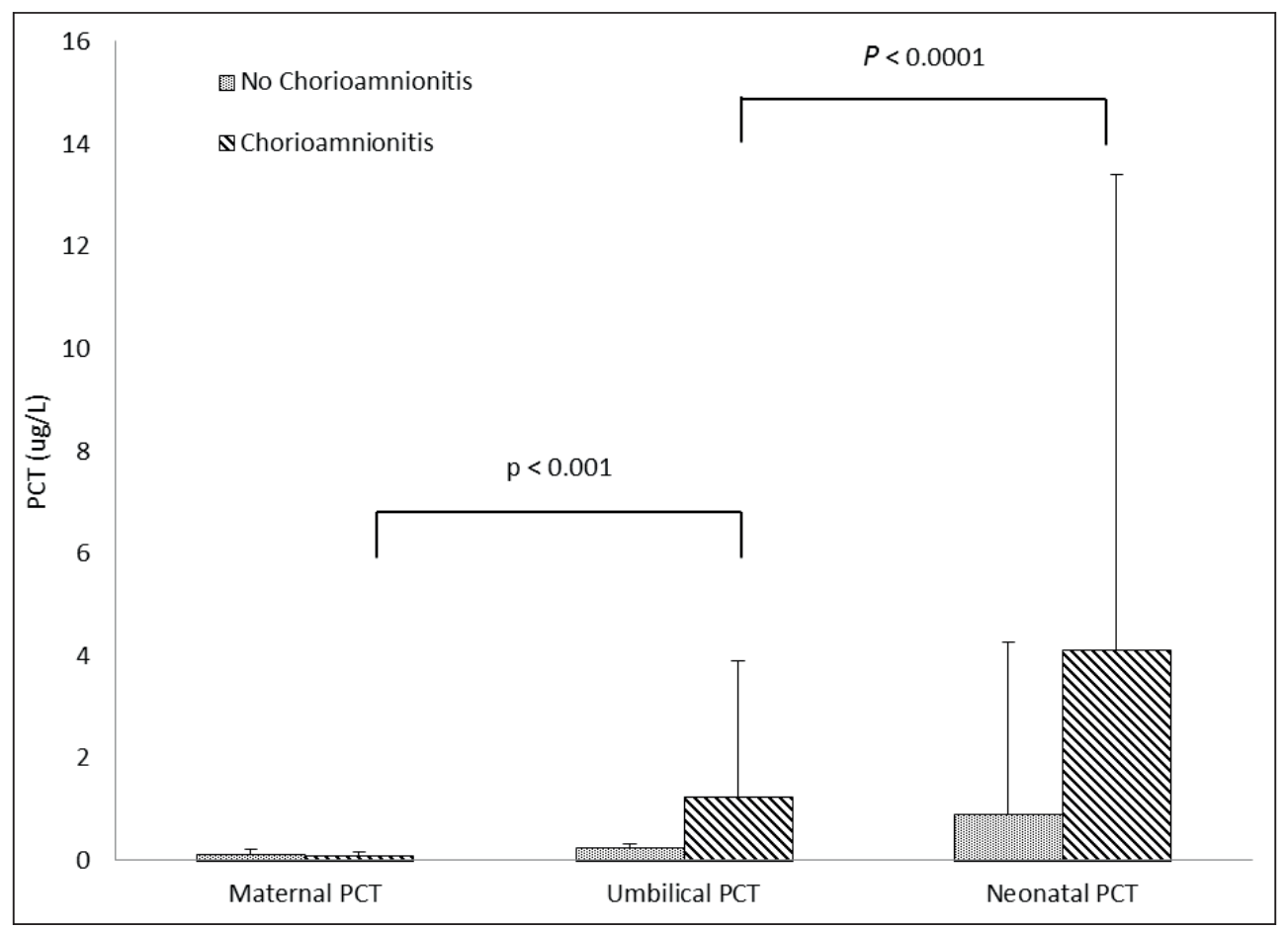

Fig. 1. The changes of PCT values in maternal, cord and neonatal blood. Mean values and positive standard deviations are shown by study group ("no chorioamnionitis" versus "chorioamnionitis"). Statistically significant difference $P<0.05$.

\section{Chorioamnionitis and neonatal outcome}

We found a strong association between chorioamnionitis and the occurrence of early onset sepsis. Preterm infants following chorioamnionitis had delayed postnatal transition and more frequent administration of antibiotics. There were no differences in other neonatal parameters. Neonatal morbidity and mortality are shown in Table 2.

\section{DISCUSSION}

Despite the progress in perinatal medicine, histological chorioamnionitis (HCA) remains a leading cause of preterm birth. Rapid diagnosis and management of HCA are essential ${ }^{9-11}$. Unfortunately, clinical signs of HCA and microbiological assessment of the amniotic fluid appear to be unreliable diagnostic critera ${ }^{2,3}$.

We investigated the role of procalcitonin (PCT) measurement in diagnosing HCA. Unfortunately, no significant difference in the maternal PCT values was found between the HCA positive and negative group. This finding is in accord with some recently published data showing that measurement of PCT in maternal blood is unreliable in the clinical diagnosis of chorioamnionitis ${ }^{2}$. Despite conflicting evidence, our study showed significant elevation in maternal WBC and CRP in the chorioamnionitis positive group ${ }^{12,13}$.

In our study, PCT was elevated in umbilical cord

Table 2. Neonatal outcome in chorioamnionitis positive and chorioamnionitis negative groups. Statistically significant difference $P<0.05$ (expressed between "no chorioamnionitis" and "chorioamnionitis" group).

\begin{tabular}{lccc}
\hline & Chorioamnionitis & No chorioamnionitis \\
& $\mathrm{n}=28(\%)$ & $P(\%)$ & 0.35 \\
\hline Gestational age (weeks), mean \pm SD & $28.5 \pm 2.5$ & $29.1 \pm 2.4$ & $1266 \pm 351$ \\
Birth weight (g), mean \pm SD & $1188 \pm 402$ & $8.5 \pm 1.0(8.2-8.8)$ & 0.01 \\
Apgar at 5 min mean \pm SD (95\% CI) & $7.9 \pm 1.1(7.5-8.4)$ & $32(51)$ & 0.12 \\
Respiratory distress syndrome, n & $20(71)$ & $3(5)$ & 0.0001 \\
Early onset sepsis, n & $10(36)$ & $31(49)$ & 0.05 \\
Use of antibiotics, n & $20(71)$ & $13(21)$ & 0.64 \\
Patent ductus arteriosus, n & $7(25)$ & $3(5)$ & 0.15 \\
Intraventricular haemorrhage grade III and IV, n & $3(10)$ & $10(16)$ & 0.30 \\
Bronchopulmonary dysplasia, n & $7(25)$ & $2(3)$ & 0.39 \\
Necrotizing enterocolitis, n & $2(7)$ & $2(3)$ & 0.39 \\
Periventricular leukomalacia, n & $2(7)$ & $62(98)$ & 0.56 \\
Survival, n & $27(96)$ & & \\
\hline
\end{tabular}


blood at the time of delivery in the HCA group. This is in accordance with some published data $^{2,3}$. Howman et al observed a modest increase of inflammatory markers in the maternal and cord blood in pregnancies complicated by histological chorioamnionitis ${ }^{2}$. Su et al found PCT to be a relatively good rule-in and rule-out marker for prediction of neonatal sepsis ${ }^{3}$. One study reported elevated cord blood levels of PCT as an independant risk factor for mortality in preterm populations less than 33 weeks gestation $^{14}$.

We confirmed significantly elevated PCT in the infants of mothers with HCA. These newborns also had increased risk for early-onset sepsis and delayed postnatal adaptation. Moreover, there was an insignificant increase in the incidence of respiratory distress syndrome (RDS) and bronchopulmonary dysplasia (BPD). Park et al associated mild to moderate HCA in preterm population with a decrease in RDS. We did not find such association perhaps due to the lack of appropriate number of patients ${ }^{14,15}$.

The strength of our study is the measurement of PCT in mother-infant pair which allowed us to evaluate the PCT changes in maternal, placental (umbilical) and neonatal circulation. Serial PCT measurements revealed that PCT values were significantly different and varied according to HCA presence or absence. Undetectable PCT values in maternal circulation in combination with positive PCT values in umbilical cord blood as well as the neonatal circulation support the hypothesis that PCT is produced by fetal tissues as a result of the fetal inflammatory response syndrome during chorioamnionitis ${ }^{16}$.

There is an apparent gradual rise of PCT in the particular compartments in mother-infant pairs with histological CHA. Coupled with other laboratory markers of infection, neonatal serial PCT measurements in at-risk infants can be quite a strong predictor of their moribidties, specifically early-onset sepsis ${ }^{15}$.

There are several limitations to the study: the relatively small size and unbalanced patient groups (28 mother-infant pairs in CHA-positive group and 63 in CHA-negative group), the data were analysed retrospectively. Maternal PCT values were obtained at various timepoints within 24 hours before delivery, which may have an impact on the comparison of maternal and cord blood values. PCT can also be increased in non-infected infants with other complications (intracranial haemorrhage, respiratory distress syndrome and perinatal asphyxia) ( ref. $^{2}$ ). Furthermore, PCT shows a physiological increase in the first few days of life, which complicates the interpretation of results ${ }^{3}$.

\section{CONCLUSION}

This study confirmed the helpful role of PCT measurement in cord and neonatal blood in the presence of proven histological chorioamnionitis. The measurement of PCT in mothers' blood does not seem useful in the diagnosis of subclinical chorioamnionitis. More studies are needed to confirm the possible release of PCT from fetus during chorioamnionitis.
Acknowledgement: This study was supported by the Research Project PRVOUK 32, Charles University, Prague.

Author contributions: ZS, JF: these authors contributed equally to this work - substantial contributions to conception and design, acquisition, analysis and interpretation of the data; PK: drafted and reviewed the article, performed data mining; LK: final approval.

Conflict of interest statement: The authors state that there are no conflicts of interest regarding the publication of this article.

\section{REFERENCES}

1. Galinsky R, Polglase GR, Hooper SB et al. The consequences of chorioamnionitis: preterm birth and effects on development. J Pregnancy 2013; Article ID 412831

2. Howman RA, Charles AK, Jacques A, Doherty DA, Simmer K, StrunkT, Richmond PC,Cole CH, Burgner DP. Inflammatory and haematological markers in the maternal, umbilical cord and infant circulation in histological chorioamnionitis. PLoS One 2012;7(12):e51836.

3. Su H, Chang SS, Han CM, Wu KY, Li MC, Huang CY, Lee CL, Wu JY, Lee CC. Inflammatory markers in cord blood or maternal serum for early detection of neonatal sepsis-a systemic review and meta-analysis. J Perinatol 2014;34(4):268-74.

4. Spinillo A, lacobone AD, Calvino IG, Alberi I, Gardella B. The role of the placenta in feto-neonatal infections. Early Hum Dev 2014,90(S1):S7-9.

5. Thornburg LL, Queenan R, Brandt-Griffith B, Pressman EK. Procalcitonin for prediction of chorioamnionitis in preterm premature rupture of membranes. J Matern Fetal Neonatal Med 2016;29(13):2056-61.

6. Chiesa C, Panero A, Rossi N, Stegagno M, De Giusti M, Osborn JF, Pacifico L. Reliability of procalcitonin concentrations for the diagnosis of sepsis in critically ill neonates. Clin Infect Dis 1998;26(3):664-72.

7. Gibbs RS, Blanco JD, St Clair PJ, Castaneda YS. Quantitative bacteriology of amniotic fluid from women with clinical intra-amniotic infection at term. J Infect Dis 1982;145(1):1-8.

8. Horbar JD. The Vermont Oxford Network: evidence-based quality improvement for neonatology. Pediatrics 1999;103(1):350-9.

9. Nasef $N$, Shabaan AE, Schurr P, laboni D, Choudhury J, Church $P$, Dunn MS. Effect of clinical and histological chorioamnionitis on the outcome of preterm infants. Am J Perinatol 2013;30(1):59-68.

10. Kim CJ, Romero R, Chaemsaithong P, Chaiyasit N, Yoon BH, Kim YM. Acute chorioamnionitis and funisitis: definition, pathologic features, and clinical significance. Am J Obstet Gynecol 2015;213(S4):S29-52.

11. Fishman SG, Gelber SE. Evidence for the clinical management of chorioamnionitis. Semin Fetal Neonatal Med 2012;17(1):46-50.

12. Trochez-Martinez RD, Smith P, Lamont RF. Use of C-reactive protein as a predictor of chorioamnionitis in preterm prelabour rupture of membranes: a systematic review. BJOG 2007;114(7):796-801.

13. Stepan $M$, Cobo T, Musilova I, Hornychova $H$, Jacobsson B, Kacerovsky M. Maternal Serum C-Reactive Protein in Women with Preterm Prelabor Rupture of Membranes. PLoS One 2016;11(3):e0150217.

14. Lautridou A, Ancel PY, Launay E, Denizot S, Orsonneau JL, Roze JC, Gras-Le Guen C. Umbilical cord blood procalcitonin as a risk factor for mortality in very premature infants. Eur J Clin Microbiol Infect Dis 2012;31(9):2407-12.

15. Altunhan H, Annagur A, Ors R, Mehmetoglu I. Procalcitonin measurement at 24 hours of age may be helpful in the prompt diagnosis of early-onset neonatal sepsis. International Journal of Infectious Diseases 2011;15:e854-e858.

16. Linscheid P, Seboek D, Nylen ES, Langer I, Schlatter M, Keller U, Becker KL, Müller B. In vitro and in vivo calcitonin-I gene expression in parenchymal cells: a novel product of human adipose tissue. Endocrinol 2003;144,5578-84. 